\title{
AVALIAÇÃO DA PREVALÊNCIA DE DELIRIUM EM UMA UNIDADE DE TERAPIA INTENSIVA PÚBLICA
}

Juliana Bessa Martins ${ }^{1}$, Adriana Alves dos Santos ${ }^{1}$, Luis Joeci Jacques de Macedo Júnior ${ }^{1}$, Carolina Chitolina Eberle ${ }^{1}$

Objetivo: Conhecer a prevalência do delirium em uma Unidade de Terapia Intensiva Clínica e Cirúrgica pública. Metodologia: Abordagem quantitativa descritiva de corte transversal, com pacientes de 18 anos ou mais internados em um hospital público de Porto Alegre-RS, com a utilização da escala Confusion Assessment Method in a Intensive Care Unit . Resultados: A prevalência do delirium foi de $36 \%$, em uma amostra de 335 pacientes entrevistados. Apresentaram relação com o delirium, com significância estatística: média de idade dos pacientes, a mediana de dias de internação hospitalar e em UTI, tipo de UTI, motivo de internação, doença neurológica anterior, uso de sedação, ventilação mecânica e utilização de haloperidol. Conclusões: Este estudo conclui que o delirium apresentou prevalência de $36 \%$, demonstrando que esta síndrome apresenta prevalência significante em Unidade de Terapia Intensiva, portanto, o reconhecimento de seus sinais e sintomas é fundamental para a sua prevenção, aliada a aplicação de escalas de rastreio.

Descritores: Delirium; Unidades de Terapia Intensiva; Enfermagem.

\section{EVALUATION OF THE PREVALENCE OF DELIRIUM IN AN PUBLIC INTENSIVE CARE UNIT}

Objective: To know the prevalence of delirium in a Public Intensive Care Clinic and Surgical Unit. Methodology: Descriptive quantitative cross-sectional approach with patients aged 18 years or more admitted to a public hospital in Porto Alegre, Brazil, using the Confusion Assessment Method in the Intensive Care Unit (CAM-ICU). Results: The prevalence of delirium was 36\%, in a sample of 335 patients interviewed. The mean age of the patients, median days of hospital stay and ICU, type of ICU, reason for hospitalization, previous neurological disease, use of sedation, mechanical ventilation and use of haloperidol were statistically significant. Conclusions: This study concludes that delirium presented a prevalence of $36 \%$, demonstrating that this syndrome presents a significant prevalence in the Intensive Care Unit, therefore, the recognition of its signs and symptoms is fundamental for its prevention, allied to the application of screening scales.

Descriptors: Delirium; Intensive Care Units; Nursing.

\section{EVALUACIÓN DE LA PREVALENCIA DE DELIRIUM EN UNA UNIDAD DE TERAPIA INTENSIVA PÚBLICA}

Objetivo: Conocer la prevalencia del delirium en una Unidad de Terapia Intensiva Clínica y Quirúrgica pública. Metodologia: En el presente trabajo se analizaron los resultados obtenidos en el análisis de los resultados obtenidos en el estudio.

Resultados: La prevalencia del delirium fue del 36\%, en una muestra de 335 pacientes entrevistados. En la mayoría de los casos, se observó un aumento en la incidencia de la enfermedad de Chagas en el momento de la intervención, Conclusiones: Este estudio concluye que el delirium presentó prevalencia del $36 \%$, demostrando que este síndrome presenta prevalencia significante en Unidad de Terapia Intensiva, por lo tanto, el reconocimiento de sus signos y sintomas es fundamental para su prevención, aliada a la aplicación de escalas de rastreo.

Descriptores: Delirium; Unidades de Terapia Intensiva; Enfermería. 


\section{INTRODUÇÃO}

Delirium é uma sindrome de natureza aguda, caracterizada pela flutuação de alterações no nível de consciência, atenção e percepção. Apresenta como manifestações clínicas: diminuição da atenção e alterações das funções cognitivas, entre elas percepção, memória, orientação e raciocínio, além de comprometimento do ciclo sono-vigília e transtornos emocionais, como depressão, ansiedade, medo, irritabilidade, euforia e apatia ${ }^{(1.2)}$.

Frequentemente, manifestam-se nos pacientes graves internados em Unidade de Terapia Intensiva (UTI) devido a fatores de risco, tais como idade elevada, uso de ventilação mecânica, submissão a procedimentos invasivos, interrupções dos ciclos de sono, hipertensão arterial sistêmica, etilismo, distúrbios metabólicos, acidose, intervenções cirúrgicas e uso de drogas, como a morfina ${ }^{(3)}$.

O surgimento de delirium é muito comum em pacientes críticos, e sua incidência pode chegar a $89 \%$, estando associada à alta mortalidade, ao aumento do tempo de internação em UTI e de ventilação mecânica, além de déficit funcional e cognitivo em longo prazo(4). Sua prevalência em UTI varia conforme a população estudada e pode ser de até $80 \%$ em pacientes submetidos à ventilação mecânica ${ }^{(5)}$. O conhecimento e a avaliação física e psíquica do paciente são importantes para um diagnóstico conciso, tratamento adequado e a busca de fatores de risco para a prevenção ${ }^{(6)}$. A escala mais utilizada para sua identificação é a Confusion Assessment Method in a Intensive Care Unit (CAM-ICU), que foi criada especificamente para utilização em UTI's(7) e verifica quatro questões: flutuação do estado mental, inatenção, pensamento desorganizado e nível de consciência alterado ${ }^{(7.8)}$.

A qualificação da equipe em reconhecer precocemente o delirium, bem como o conhecimento de sua prevalência em UTI, contribuirá para o planejamento de intervenções, melhoria da assistência e diminuição das complicações causadas por essa sindrome; sendo assim, o presente estudo tem como objetivo descrever a prevalência do delirium em uma UTI Clínica e Cirúrgica pública.

\section{METODOLOGIA}

\section{Tipo de estudo}

Estudo quantitativo descritivo de corte transversal.

\section{Participantes da pesquisa}

A população do estudo foi composta por pacientes de ambos os sexos, internados na UTI no período de Abril a Outubro de 2017 e que estiveram de acordo com os critérios de inclusão deste estudo. O tamanho calculado da amostra com nível de confiança de 95\%, contabilizando 5\% de margem de erro, totalizam 335 pacientes que foram entrevistados.

Os critérios de inclusão deste estudo foram: ter 18 anos ou mais, estar internado na UTI por pelo menos 48 horas no momento da coleta dos dados, possuir capacidade de comunicação, e também ausência de sedação profunda mensurada pela Escala de Agitação e Sedação de Richmond (RAAS), com escore igual ou superior a -3 .

\section{Local do estudo}

Este estudo foi realizado em uma UTI de um hospital público de Porto Alegre-RS.

\section{Coleta de dados}

Utilizou-se como instrumento um formulário estruturado, composto por questões destinadas a mensurar variáveis sociodemográficas, epidemiológicas e clínicas, elaborado pela pesquisadora. Estes dados foram consultados através do prontuário do paciente internado. Também foi utilizado o instrumento CAM-ICU para rastreamento; a aplicação dessa escala dura menos de dez minutos e é realizada à beira leito do paciente. Para o emprego dessa ferramenta, foram necessárias escalas auxiliares: A escala de agitação e sedação de Richmond (RASS) e o Teste de Exame de Atenção. Ressalta-se que neste estudo foi utilizado apenas o Teste de Exame de Atenção com componente auditivo, considerando o nosso perfil de paciente, na grande maioria de idosos com algum tipo de dificuldade visual.

A variável dependente deste estudo foi o resultado da aplicação da escala CAM-ICU e as variáveis independentes foram: sociodemográficas (sexo, idade, escolaridade), epidemiológicas (tempo de internação hospitalar, tempo de internação em UTI, tipo de UTI internado e motivo de internação), clínicas (comorbidades associadas, doença neurológica anterior, uso de sedação, ventilação mecânica e uso de haloperidol).

\section{Procedimentos de análise dos dados}

Os dados coletados foram digitados em uma planilha eletrônica estruturada no programa Microsoft Excel versão $2010 \AA$, sob forma de dupla digitação no sentido de promover a eliminação de erros e garantir a confiabilidade na compilação dos dados; para verificação da consistência interna e análise estatística, foi utilizado o programa SPSS. A análise dos resultados foi descritiva e utilizado o teste qui-quadrado de Pearson, teste exato de Fisher ou teste U de Mann-Whitney, quando necessário, para analisar os fatores de risco categóricos e teste T de Student para as variáveis contínuas.

\section{Procedimentos éticos}

O presente estudo foi aprovado pelo Comitê de Ética em Pesquisa sob protocolo número 130305/2016, seguindo 
todos os preceitos da Resolução do Conselho Nacional de Saúde $n^{\circ} 466 / 2012^{(9)}$, e conduzido de acordo com os padrões éticos exigidos.

\section{RESULTADOS}

Analisando as características dos pacientes internados na UTI, durante o período deste estudo, do total de 335 pacientes participantes, 193 (58\%) pertenciam ao sexo feminino e 142 (42\%) ao sexo masculino. Sua idade variou de 18 a 92 anos, com média de idade 62 anos (DP +- 15,67). A respeito da escolaridade, a maioria, 259 (77\%) possuíam o 1으 grau. Em relação ao tipo de UTI internados, 187 participantes (56\%) estavam internados em UTI clínica, e 148 (44\%) em UTI cirúrgica. Avaliando a prevalência do delirium, objetivo deste trabalho, podemos observar que 120 (36\%) dos pacientes apresentaram delirium segundo a escala CAM-ICU e 215 (64\%) dos pacientes apresentaram CAM-ICU negativo para delirium.

As variáveis sociodemográficas foram dividas em diferentes critérios: de acordo com o sexo, daqueles que apresentaram delirium, 65 (54\%) eram mulheres, e 55 (46\%) eram homens, não apresentando significância estatística $(p=0,341)$. No que se refere à idade, houve significância estatística ( $p=$ 0,012 ). Os pacientes que apresentaram delirium tiveram média de idade de 64,58 anos (DP +- 14,85), superior àqueles que não tiveram delirium, que tiveram média de idade de 60,18 anos (DP +- 15,92). Em relação à escolaridade, não houve significância estatística ( $p=0,167)$. Dos pacientes que apresentaram delirium, 12 (10\%) não eram alfabetizados, 87 (72\%) possuíam lo grau, 19 (16\%) possuíam 2ㅇ grau e 2 (2\%) possuíam ensino superior.

De acordo com as variáveis epidemiológicas, os pacientes que apresentaram delirium, apresentaram como mediana 20,50 dias de internação hospitalar, com significância estatística $(p=0,003)$. No que se refere aos dias de internação em UTI obtivemos como mediana 8 dias de internação, com significância estatística ( $p<0,001)$. $O$ tipo de UTI também apresentou inferência para o surgimento de delirium na UTI, 86 (72\%) dos pacientes com delirium estavam internados em UTI do tipo clínica e 34 (28\%) estavam internados em unidade cirúrgica, demonstrando maior prevalência de delirium em UTI clínica, com significância estatística $(p<0,001)$.

Em relação ao motivo de internação, obteve-se significância estatística ( $p<0,001$ ). Dos pacientes que apresentaram delirium, 26 (22\%) internaram por sepse e 39 (32\%) tiveram como motivo de internação a insuficiência ventilatória. Ao associar as comorbidades clínicas, a doença neurológica anterior apresentou relação com o delirium, 16 (13\%) haviam AVC-I prévio e 8 (7\%) possuíam epilepsia, com significância estatística ( $p=0,027$ e $p=0,005$, respectivamente).

De acordo com a tabela 1 , ao analisar o nível de sedação dos pacientes com delirium através da escala de agitação-sedação de Richmond (RASS), podemos perceber, com significância estatística ( $p<0,001)$, que os pacientes que apresentaram RASS diferente de zero, ou seja, os que apresentaram-se mais agitados ou mais sonolentos, possuem maior propensão para manifestação de delirium delirium 26 (22\%) do que os pacientes que apresentaram RASS +1 (inquietos); 31 (26\%) apresentaram RASS -1 (sonolentos); 14 (12\%) apresentaram RASS -2 (sedados levemente) e 23 (19\%) apresentaram RASS -3 (sedados moderadamente).

O uso de sedação influencia na manifestação do delirium. 28 (23\%) dos pacientes que apresentaram delirium, estavam sob efeito de alguma sedação, com significância estatística $(p<0,05)$. Os tipos de sedação que mais tiveram relação com a presença de delirium, foram: fentanil 24 (86\%) e propofol 4 (14\%), com significância estatística ( $p<0,001$ e $p=0,016$, respectivamente)

O uso de ventilação mecânica também apresentou influência sobre a manifestação do delirium. Dos pacientes que apresentaram essa condição, 62 (52\%) estavam fazendo uso de ventilação mecânica, com significância estatística $(p<0,001)$. Dos pacientes que obtiveram CAM-ICU positivo para delirium, 30 (25\%), estavam fazendo uso de Haloperidol, com significância estatística $(p<0,001)$

Tabela 1 - Distribuição das variáveis relacionadas ao tratamento, segundo a presença do delirium, de acordo com a escala de sedação de RASS, uso de sedação, tipo de sedação, uso de ventilação mecânica e uso de Haldol. Porto Alegre, RS, $2017(\mathrm{~N}=335)$.

\begin{tabular}{|ccccc|}
\hline Variável & $\begin{array}{c}\text { Com deli- } \\
\text { rium } \\
(\mathbf{N}=\mathbf{1 2 0})\end{array}$ & $\begin{array}{c}\text { Sem deli- } \\
\text { rium } \\
(\mathbf{N}=\mathbf{1 2 5})\end{array}$ & Total & Valor $\mathbf{p}$ \\
\hline Escala de RASS & & & & \\
\hline+3 & $1(0,83)$ & $0(0)$ & 1 & \\
\hline+2 & $8(6,67)$ & $0(0)$ & 8 & \\
\hline+1 & $26(21,67)$ & $3(1,40)$ & 29 & \\
\hline 0 & $17(14,17)$ & $183(85,12)$ & 200 & $<0,001^{*}$ \\
\hline-1 & $31(25,83)$ & $25(11,63)$ & 56 & \\
\hline-2 & $14(11,67)$ & $4(1,86)$ & 18 & \\
\hline-3 & $23(19,17)$ & $0(0)$ & 23 & \\
\hline Uso de sedação & & & & \\
\hline Sim & $28(23,33)$ & $8(3,72)$ & 36 & $<0,001^{*}$ \\
\hline Não & $92(76,67)$ & $207(96,28)$ & 299 & \\
\hline
\end{tabular}

Tipo de sedação

$\begin{array}{ccccc}\text { Fentanil } & 24(85,71) & 7(87,50) & 31 & <0,001^{*} \\ \text { Propofol } & 4(14,28) & 0(0) & 4 & 0,016^{*} \\ \text { Midazolam } & 5(17,85) & 3(37,5) & 8 & 0,112^{*}\end{array}$

Ventilação mecânica

$\begin{array}{ccccc}\text { Sim } & 62(51,67) & 33(15,35) & 95 & <0,001^{*} \\ \text { Não } & 58(48,33) & 182(84,65) & 290 & \end{array}$


Haloperidol

$\begin{array}{ccccc}\text { Sim } & 30(25,00) & 3(1,40) & 33 & <0,001^{*} \\ \text { Não } & 90(75,00) & 212(98,60) & 302 & \end{array}$

$\left({ }^{*}\right)$ - Teste exato de Fisher.

\section{DISCUSSÃO}

A prevalência do delirium encontrada neste estudo foi semelhante à de resultados encontrados na literatura, os quais destacam a prevalência de delirium em pacientes idosos. Chávez-Delgado et al.(10), obtiveram em seu estudo prevalência similar, de $38,3 \%$ e incidência de $11,7 \%$ de delirium entre idosos internados. Alguns estudos trazem que a prevalência desta disfunção pode chegar até $70 \%$ dependendo das características populacionais ${ }^{(11)}$. Apesar dos pacientes do sexo masculino serem mais susceptiveis ao desenvolvimento desta síndrome ${ }^{(12)}$, no presente estudo a diferença de ocorrência de delirium entre os sexos não foi estatisticamente significativa. A idade média de pacientes com delirium apresentadas neste estudo, esteve próxima a literatura, ao considerar que pacientes idosos, com 65 anos ou mais, possuem a faixa etária com maior predisposição ao delirium ${ }^{(12.13)}$.

Este estudo apresentou significância estatística ao analisar que o tempo de internação hospitalar e o tempo de internação em UTI, estão relacionados ao maior desenvolvimento de delirium. Existem evidências de que pacientes críticos que apresentam sinais e sintomas desta condição tendem a aumentar seu tempo de internação na UTI ${ }^{(8)}$. Ao associar o delirium com o tipo de UTI que o paciente estava internado, obteve-se maior relevância estatística em pacientes internados em UTI clínica. Mori et al. (2016) identificaram, em seu estudo, que o motivo de admissão hospitalar predominantemente em pacientes com delirium foi clínico $(p<0,001)^{(14)}$. Esse dado pode estar relacionado ao fato de que o paciente internado em UTI clínica comumente apresenta um número maior de patologias e tempo de internação se comparado ao internado em UTI cirúrgica.

A gravidade da doença de base é reconhecida como importante fator de risco para delirium. Dos pacientes que apresentaram delirium, 26 (22\%) internaram por sepse e 39 (32\%) tiveram como motivo de internação, na UTI, a insuficiência ventilatória. Esses motivos apresentaram relação com o desenvolvimento do delirium, possivelmente, relacionado ao maior tempo de internação e consequente aumento dos fatores de risco predisponentes ${ }^{(15)}$.

A escala de agitação-sedação de RASS é a ferramenta de avaliação da sedação mais confiável para medir a qualidade e profundidade da sedação em pacientes adultos internados em UTI. Nesse estudo, pacientes que possuíam RASS diferente de zero, ou seja, estavam mais sonolentos ou mais agitados, apresentaram propensão ao desenvolvimento de de- lirium, com significância estatística ( $p<0,001)$; essa avaliação também pode relacionar-se com o tipo de delirium desenvolvido (hipoativo, hiperativo ou misto). É de grande importância que o paciente tenha diminuição ou ausência de sedação em algum período do dia, propiciando um despertar diário, para a avaliação do delirium.

Dos pacientes que estavam sob uso de sedação, 28 (23\%) apresentaram delirium, com significância estatística $(p<0,05)$. A sedação excessiva se associa com aumento da duração da ventilação mecânica, de permanência na UTI, e aumento das taxas de mortalidade ${ }^{(4)}$. Há evidências que indicam os benzodiazepínicos como drogas associadas a maior ocorrência de delirium ${ }^{(12)}$. Diferentemente, dos pacientes que apresentaram delirium, 24 (86\%) utilizavam fentanil e o 4 (14\%) utilizavam propofol como sedativo, com significância estatística ( $p<0,001$ e $p=0,016$, respectivamente).

Corroborando com este estudo, outro autor constatou que 38,46 dos idosos com delirium utilizavam fentanil, opióide de analgesia contínua da instituição cenário da pesquisa, o que aumentou em 18 vezes a chance de desenvolver a síndrome em questão, sendo identificado que os pacientes que receberam essa medicação eram mais propensos ao desenvolvimento deste fenômeno ${ }^{(15)}$.

Há evidências atuais de que o delirium está associado com piores desfechos para pacientes criticamente enfermos, inclusive aumento da duração da ventilação mecânica, permanência hospitalar e mortalidade ${ }^{(4)}$. Este estudo está de acordo com a literatura ao afirmar que o uso de ventilação mecânica apresenta relação para os pacientes apresentarem delirium. Dos pacientes que apresentaram delirium, 62 (52\%) estavam fazendo uso de ventilação mecânica, com significância estatística $(p<0,001)$.

Autores referem que na adoção de medidas farmacológicas, o antipsicótico típico de primeira geração, o haloperidol, tem sido o fármaco de eleição utilizado para o delirium na sua forma mais hiperativa ${ }^{(16)}$. Neste estudo, dos pacientes que tiveram CAM-ICU positivo para delirium, 30 (25\%) estavam fazendo uso de haloperidol, com significância estatística $(p<0,001)$. O uso profilático do haloperidol, em pacientes com alto risco para delirium, pode reduzir complicações ${ }^{(17)}$. Porém, em um estudo prospectivo realizado com pacientes de alto risco para delirium internados por fratura de quadril, em que se utilizou, de forma profilática, o haloperidol, os resultados não mostraram redução em sua incidência ${ }^{(18)}$. O estudo Hope-ICU demonstrou que a administração rotineira de haloperidol não reduz a duração do delirium quando diagnosticado com a escala CAM-ICU, porém, este estudo demonstrou que o haloperidol auxilia na redução da agitação(19).

Como ainda há divergências na literatura sobre o uso do haloperidol, as medidas não farmacológicas apresentam-se 
como ferramenta importante para o tratamento do paciente com delirium na UTI, destacando-se: estimular a presença de acompanhantes, uso de óculos e aparelhos auditivos, acesso ao horário e às medicações que está em uso, orientação no tempo e espaço e mobilização precoce, entre outras.

Limitações do estudo

Considera-se como limitação do estudo, seu delineamento como estudo transversal, o qual não permite fazer inferências causais, pois não há como distinguir a temporalidade dos fatos de exposição e desfecho.

Contribuições do estudo para prática

Este estudo contribui com a prática da Enfermagem em terapia intensiva, pois conhecer a prevalência do delirium e identificar precocemente seus sinais e sintomas, melhoram a assistência e os cuidados prestados aos pacientes, contribuindo para melhores desfechos clínicos.

\section{CONCLUSÃO}

Os resultados deste estudo mostraram que o delirium apresentou prevalência de 36\% nos pacientes internados em UTI, mostrando que essa sindrome apresenta prevalência significante nesse ambiente. Suas causas são multifatoriais; neste estudo, a idade, mediana de dias de internação hospitalar e em UTI, tipo de UTI, motivo de internação (sepse e insuficiência ventilatória), bem como doença neurológica anterior, uso de sedação, ventilação mecânica e utilização de haloperidol, apresentaram relação com o delirium, com significância estatística.

A equipe multiprofissional, principalmente a Enfermagem, assume papel primordial na identificação de fatores de risco para o desenvolvimento do delirium, reconhecimento de sinais e sintomas, implementando medidas de prevenção não farmacológicas, modificando positivamente o ambiente para que se torne menos hostil e mais humanizado.

O enfermeiro possui atividades privativas, autonomia profissional, deveres e obrigações e vedações na atuação no gerenciamento e assistência que envolve os serviços e cuidados de enfermagem. Bem como, ele torna-se fundamental, no cuidado do paciente internado, garantindo que as escalas de avaliação que detectam o delirium à beira leito sejam aplicadas no cotidiano da avaliação dos pacientes.

Almeja-se que os resultados desse estudo possam contribuir para a melhoria do cuidado prestado aos pacientes internados em UTI que apresentem predisposição a ter delirium, além de estimular que outros estudos apresentem resultados sobre essa sindrome, com o intuito de apontar os agravos causados pela mesma, para que assim possamos planejar, prevenir e melhorar a eficiência ao atendimento a pacientes acometidos com delirium.

\section{Contribuição dos autores}

Juliana Bessa Martins, responsável por todos os aspectos do estudo, assegurando as questões de precisão ou integridade de qualquer parte do estudo. Desde a concepção do estudo, análise e interpretação dos dados a redação do artigo. Adriana Alves dos Santos, responsável pela revisão final do estudo a ser publicado. Luís Joeci Jacques de Macedo Júnior, elaboração e revisão crítica do conteúdo intelectual do estudo.Carolina Chitolina Eberle, contribuições substanciais para a concepção e desenho do estudo. 


\section{REFERÊNCIAS}

1. Barros MAM. Fiquêiredo DSTO. Fernandes MGM. Melo MDG. Neto R, Melquiades $\mathrm{J}$, et al. Delirium em idosos em unidades de terapia intensiva: revisão integrativa da literatura. J. res.: fundam. care. [Internet]. 2015 [cited 2017 Jul 18]; jul./set. va da literatura. J. res: fundam. care. [Internet]. 2015 [cited 2017 Jul 18]; jul./set.
7(3):2738-2748. Available from: http://www.seer.unirio.br/index.php/cuidadofundamental/article/view/3347/pdf_1614

2. Carvalho JLM, Almeida ARP de, Gusmao-flores D. Escalas de avaliação de delirium em pacientes graves: revisão sistemática da literatura. Rev. bras. ter. intensiva [Internet]. 2013 [cited 2017 Jul 23]:25(2):148-154. Available from: http://www. scielo.br/pdf/rbti/v25n2/v25n2al3.pdf

3. Faria RSB, MORENO RP. Delirium na unidade de cuidados intensivos: Uma realidade subdiagnosticada. Rev. bras. ter. intensiva [Internet]. 2013 [cited 2017 Jul 27] 25(2):137-147. Available from: http://www.scielo.br/pdf/rbti/v25n2/v25n2al2.pdf

4. Mori S, Takeda JRT, Carrara FSA, Cohrs CR, Zanei SSV. Whitaker IY. Incidência e fatores relacionados ao delirium em Unidade de Terapia Intensiva. Rev Esc Enferm USP [Internet]. 2016 [cited 2017 Jul 27];50(4):587-593. Available from: http://www. scielo.br/pdf/reeusp/v50n4/pt_0080-6234-reeusp-50-04-0587.pdf

5. Tanaka LM, Salluh JI, Dal-Pizzol F, Barreto BB, Zantieff R, Tobar E, et al. Delirium em pacientes na unidade de terapia intensiva submetidos à ventilação não invasiva. Rev Bras Ter Intensiva [Internet]. 2015 [cited 2017 Jul 30]:27(4):360-368. Available from: http://www.scielo.br/pdf/rbti/v27n4/0103-507X-rbti-27-04-0360.pdf

6. Boogaard MVD, Pickkers P. Slooter A JC, Kuiper MA. Spronk PE, Voort PHJVD et al. Development and Validation of PREDELIRIC (PREdiction of DELIRium in ICu patient) delirium prediction model for intensive care patients: observational multicenter study. BMJ [Internet]. 2012 [cited 2017 Jun 30]:344:e420. Available from: https://www.ncbi.nlm.nih.gov/pmc/articles/PMC3276486/

7. Hipp MD. Elly EW. Pharmacological and nonpharmacological management of delirium in critically ill patients. Neurotherapeutics [Internet]. 2012 [cited 2017 delirium in critically ill patients. Neurotherapeutics [Internet]. 2012 [cited 2017
Jul 15] 9(1):158-75. Available from: https://www.ncbi.nlm.nih.gov/pmc/articles/ Jul 15] 9(1):158
PMC3271151/

8. Pincelli EL, Waters C, Hupsel ZN. Ações de enfermagem na prevenção do delirium em pacientes na Unidade de Terapia Intensiva. Arq Med Hosp Fac Cienc Med Santa Casa São Paulo [Internet]. 2015 [cited 2017 Ago 15];60:131-9. Available from: http://arquivosmedicos.fomsantacasasp.edu.br/index.php/AMSCSP/article/viewFile/143/592

9. Brasil. Conselho Nacional de Saúde. Resolução n 466 , 2012. Diretrizes e Normas regulamentadoras de pesquisa envolvendo seres humanos. Brasilia, 13 jun. 2013. Seção 1 p. 59 [Internet]. 2013 [cited 2017 Ago 15]. Available from: http://bvsms. saude.gov.br/bvs/saudelegis/cns/2013/res0466_12_12_2012.html

10. Chávez-Delgado ME, Virgen-Enciso N. Peréz-Guzmán J, Celis-de-la-Rosa A Castro-Castañeda S. Detection of delirium in hospitalized elderly patients using Castro-Castaneda S. Detection of delirium in hospitalized elderly patients using
the confusion assessment method. Rev Med Inst Mex Seguro Soc [Internet] 2007 [cited 2017 Ago 15]; Jul-Aug:45(4):321-8. Available from: https://www.ncbi.nlm.nih. gov/pubmed/17949569
11. Dessap AM, Roche-campo F, Launay JM.: Charles-Nelson A, Katsahian S. Brun-Buisson $C$ et al Delirium and circadian rhythm of melatonin during waning from mechanical ventilation: an ancillary study of wearing trial. Chest [Internet] 2015 [cited 2017 Ago 15]; Nov;148(5):1231-1241. Available from: https://www.ncbi.nlm.nih. gov/pubmed/26158245

12. Luna AA. Delirium em terapia intensiva - um estudo retrospectivo. Dissertação (Mestrado em Enfermagem) - Universidade Federal do Estado do Rio de Janeiro. Rio de Janeiro, 2013.

13. Mesa P, Previgliano IJ, Altez S, Favretto S, Orellano M, Lecor C, et al. Delirium em uma unidade de terapia intensiva latino-americana. Estudo prospectivo em coorte em pacientes em ventilação mecânica. Rev. bras. ter. intensiva [Internet] 2017 [cited 2017 Jul 27]:(3):337-345. Available from: < http://www.scielo.br/pdf/ rbti/v29n3/0103-507X-rbti-29-03-0337.pdf>

14. Mori S, Takeda JRT, Carrara FSA, Cohrs CR, Zanei SSV, Whitaker IY, Incidência e fatores relacionados ao delirium em Unidade de Terapia Intensiva. Rev Esc Enferm USP [Internet]. 2016 [cited 2017 Ago 25]:50(4):587-593. Available from: http://www. scielo.br/pdf/reeusp/v50n4/pt_0080-6234-reeusp-50-04-0587.pdf

15. Barros MAA de. Delirium em idosos criticamente enfermos: um estudo utilizando a ferramenta CAM-ICU. Dissertação (mestrado). UFPB. João Pessoa, 2014.

16. Page VJ, Casarin A. Uso de antipsicóticos para tratamento do delirio na unidade 16. Page $V$ J , Casaria intensiva. Rev Bras Ter Intensiva [Internet]. 2014 [cited 2017 Ago 17] de terapia intensiva. Rev Bras Ter Intensiva [Internet]. 2014 [cited 2017 Ago 17]
26(2):86-88. Available from: http://www.scielo.br/pdf/rbti/v26n2/0103-507X-rbti-26-02-0086.pdf

17. Ribeiro SCL, Nascimento ERP, Lazzari DD, Jung W. Boes AA, Bertoncello KC. Conhecimento de enfermeiros sobre delirium no paciente crítico: discurso do sujeito coletivo. Texto Contexto Enferm. Florianópolis [Internet], 2015 [cited $2017 \mathrm{Jul}$ 27]: Abr-Jun; 24(2): 513-20. Available from: http://www.scielo.br/pdf/tce/v24n2/ pt_0104-0707-tce-24-02-00513.pdf

18. Vochteloo A J, Moerman S, Van der Burg BLSB, Boo M, Vries MRde, Niesten $\mathrm{DD}$, et al.Delirium risk screening and haloperidol prophylaxis program in hip fracture patients is a helpful tool in identifying high-risk patients, but does not reduce the incidence of delirium. BMC Geriatr, [Internet] 2011 [cited 2017 Ago 231:11(39). Available from: https://bmcgeriatr.biomedcentral.com/articles/10.1186/ 1471-2318-11-39\#Abs1

19. Page VJ, Ely EW, Gates S. Zhao XB, Alce T. Shintani A, et al. Effect of intravenous haloperidol on the duration of delirium and coma in critically ill patients (Hope-ICU): a randomised, double-blind, placebo-controlled trial. Lancet Respir Med. [Internet] 2013 [cited 2017 Ago 23] Sep; 1(7): 515-523. Available from: https://www. ncbi.nlm.nih.gov/pubmed/24461612

20. Andrade SJde, Schmitt MD, Schittler ML, Ferreira A, Ruoff, AB, Piccoli T. Configuração da gestão do Cuidado de enfermagem no Brasil: uma análise documental. Enferm Foco [ Internet] 2019; [cited 2019 Ago 9] 10(1):127-133. Available form: http://revista.cofen.gov.br/index.php/enfermagem/article/view/1926/508 